\title{
Overexpressed PLAU and its potential prognostic value in head and neck squamous cell carcinoma
}

\author{
Zhexuan Li ${ }^{1,2,3}$, Changhan Chen ${ }^{1,2,3}$, Juncheng Wang ${ }^{1,2,3}$, Ming Wei ${ }^{1,2,3}$, Guancheng Liu ${ }^{1,2,3}$, Yuexiang Qin ${ }^{1,2,3}$, Li $^{1,2,3}$ \\ She ${ }^{1,2,3}$, Yong Liu ${ }^{1,2,3,4}$, Donghai Huang ${ }^{1,2,3,4}$, Yongquan Tian ${ }^{1,2,3}$, Gangcai Zhu ${ }^{\text {Corresp., }}$, Xin Zhang $^{1,2,3,4}$ \\ ${ }^{1}$ Department of Otolaryngology-Head and Neck Surgery, The Xiangya Hospital,Central South University, China, Hunan, China \\ 2 Otolaryngology Major Disease Research Key Laboratory of Hunan Province, Changsha, Hunan, China \\ 3 Clinical Research Center for Pharyngolaryngeal Diseases and Voice Disorders in Hunan Province, Changsha, Hunan, China \\ 4 National Clinical Research Center for Geriatric Disorders, Changsha, Hunan, China \\ 5 Department of Otolaryngology-Head and Neck Surgery, The Second Xiangya Hospital, Central South University, Changsha, Hunan, China \\ Corresponding Author: Gangcai Zhu \\ Email address: qianhudoctor@csu.edu.cn
}

Background. Metastasis is a major event for survival and prognosis in patients with head and neck squamous cell carcinomas (HNSCC). A primary cause of metastasis is the proteolytic degradation of the extracellular matrix (ECM). The plasminogen activator urokinase (PLAU) is involved in the transformation of plasminogen to plasmin leading to hydrolyzation of ECM-related proteins. However, the role of PLAU expression in HNSCC is unclear and worth to be investigated. Methods. PLAU expression profiles and clinical parameters from multiple HNSCC datasets were used to investigate the relationship of PLAU expression and HNSCC survival. GO and PPI network were established on PLAUrelated downstream molecular. The stroma score was deconvoluted for analysis of PLAU's association with the immune environment. ROC analysis was applied to show the performance of PLAU in predicting HNSCC prognosis. Results. PLAU mRNA was significantly elevated, as opposed to its methylation, in HNSCC tumor samples over normal specimens (all $p<0.01$ ). Univariate and multivariate cox analysis showed PLAU could be an independent indicator for HNSCC prognosis. Combining with neck lymph node status, the AUC of PLAU in predicting 5-years overall survival reached to 0.862 . GO enrichment analysis showed the major biological process (extracellular matrix organization and the P13K-Akt signaling pathway) may involve to the possible mechanism of PLAU's function on HNSCC prognosis. Furthermore, PLAU expression was positively correlated with stroma cell score, M1 type macrophages, and negatively associated with CD4 + T cell, Tregs cell, and follicular helper T cell. Conclusions:PLAU might be an independent biomarker for predicting outcomes of HNSCC patients. The elevated expression of PLAU was associated with HPV positivity and neck node status. PI3K-Akt pathway and aberrant proportions of immune cells might underly the mechanism of PLAU's oncogene role in HNSCC. 
1 Overexpressed PLAU and its potential prognostic value in

\section{2 head and neck squamous cell carcinoma}

3 Zhexuan $\mathrm{Li}^{1,2,3}$, Changhan Chen ${ }^{1,2,3}$, Juncheng Wang ${ }^{1,2,3}$, Ming Wei ${ }^{1,2,3}$, Guancheng Liu ${ }^{1,2,3}$,

4 Yuexiang Qin ${ }^{1,2,3}$, Li She ${ }^{1,2,3}$, Yong Liu ${ }^{1,2,3,4}$, Donghai Huang ${ }^{1,2,3,4}$, Yongquan Tian ${ }^{1,2,3}$, Gangcai

$5 \mathrm{Zhu}^{5 *}$, Xin Zhang ${ }^{1,2,3,4}$

7 1. Department of Otolaryngology-Head and Neck Surgery, The Xiangya Hospital, Central South

8 University, Changsha 410008, China

9 2. Otolaryngology Major Disease Research Key Laboratory of Hunan Province, 87 Xiangya

10 Road, Changsha, Hunan 410008, China.

11 3. Clinical Research Center for Pharyngolaryngeal Diseases and Voice Disorders in Hunan

12 Province, 87 Xiangya Road, Changsha, Hunan 410008, China

\section{Road, Changsha, Hunan 410008, China}

5. Department of Otolaryngology-Head and Neck Surgery, The Second Xiangya Hospital, Central South University, Changsha 410010, China.

\section{Corresponding Author:}

Gangcai Zhu

Yuanjialing Shangquan, Yuhua District, Changsha, China

E-mail: qianhudoctor@csu.edu.cn

\section{Abstract}

Background. Metastasis is a major event for survival and prognosis in patients with head and neck squamous cell carcinomas (HNSCC). A primary cause of metastasis is the proteolytic degradation of the extracellular matrix (ECM). The plasminogen activator urokinase (PLAU) is involves in the transformation of plasminogen to plasmin leading to hydrolyzation of ECM related proteins. However, the role of PLAU expression in HNSCC is unclear and worth to be investigated.

Methods. PLAU expression profiles and clinical parameters from multiple HNSCC datasets were used to investigate the relationship of PLAU expression and HNSCC survival. GO and PPI network were established on PLAU-related downstream molecular. The stroma scores were deconvoluted for analysis of PLAU's association with the immune environment. ROC analysis was applied to show the performance of PLAU in predicting HNSCC prognosis. Results. PLAU mRNA was significantly elevated, as opposed to its methylation, in HNSCC tumor samples over normal specimens (all $\mathrm{p}<0.01$ ). Univariate and multivariate cox analysis showed PLAU could be an independent indicator for HNSCC prognosis. Combining with neck lymph node status, the AUC of PLAU in predicting 5-years overall survival reached to 0.862. GO enrichment analysis 
showed the major biological process (extracellular matrix organization and the P13K-Akt signaling pathway) may involve to the possible mechanism of PLAU's function on HNSCC prognosis. Furthermore, PLAU expression was positively correlated with stroma cell score, M1 type macrophages, and negatively associated with CD4 + T cell, Tregs cell, and follicular helper T cell.

Conclusions. PLAU might be an independent biomarker for predicting outcomes of HNSCC patients. The elevated expression of PLAU was associated with HPV positivity and neck node status. PI3K-Akt pathway and aberrant proportions of immune cells might underly the mechanism of PLAU's oncogene role in HNSCC.

Keywords. The plasminogen activator urokinase (PLAU), head and neck squamous cell carcinomas (HNSCC), The Cancer Genome Atlas (TCGA), Gene Expression Omnibus (GEO),bioinformatics analysis, Survival

\section{Introduction}

Head and neck squamous cell carcinomas (HNSCC) are among the most aggressive malignancies and over $50 \%$ of patients present with locally advanced or metastatic disease(Torre et al. 2015). More than 830,000 patients are diagnosed and over 430,000 patients die from this disease worldwide annually (Cramer et al. 2019). This disease is characterized by low survival rates, high recurrence rates, and/or regional lymph node that become metastatic (Siu et al. 2019). Although the examination and treatment has been improving in recent decades, the overall 5-year survival rate of HNSCC patients doesn't increase remarkably (Yang et al. 2019). Prognosis prediction is crucial for physicians to offer consultants and personized treatment. However, clinical parameters such as TNM classification are the main sources physicians generally relying on for predicting patient outcome and making therapeutic decision, which is inaccurate in many situation(Kowalski et al. 2005; Moertel et al. 1995).It's well accepted that molecular biomarkers may facilitate the prognosis prediction for SCCHN patients(Kang et al. 2015; Leemans et al. 2018). Currently, there is no matured biomarkers is approved for HNSCC prognosis prediction. Therefore, it is expected and worth that the identification of novel biomarkers assisting with patient care and survival improvement.

Metastasis is one of the major events leading to unfavorable survival time for HNSCC patients (Chen et al. 2018). The mechanism of HNSCC metastasis is unknown, accumulated evidence show that ECM reconstruction may involve providing a physical and biochemical niche for humor cell metastasis(Hanahan \& Weinberg 2011; Murphy \& Courtneidge 2011).

PLAU belonging to the S1 serine peptidase of Clan PA, also named Urokinase-type plasminogen activator (uPA) is a proteinase involving in the transformation of plasminogen to plasmin(Ai et al. 2020). And it could hydrolyze ECM remodeling related proteins and activates growth factors(Danø et al. 2005). Some studies report that the expression level of PLAU is significantly correlated to tumor cell lymph node and distant organ metastasis (Gutierrez et al. 2000). Emerging evidence implies that PLAU plays a critical role in the initiation and development of various cancers including breast cancer, colorectal cancer, and esophageal cancer( $\mathrm{Li}$ et al. 2017; Lin et al. 2019; Novak et al. 2019).However, the role of PLAU needs to be explored further in 
78 HNSCC. Here, we applied multiple datasets to evaluate the increased expression of PLAU in

HNSCC tumor samples as compared to adjacent tissues and confirm it as an independent prognosis predictor of HNSCC patients in different angles and levels. The co-expression network and scores of tumor immune microenvironment were established and analyzed in this study as well, which could be interpreted to possible mechanism of PLAU's role in HNSCC patients.

\section{Materials \& Methods}

\section{Data collection and normalization}

GSE25099 from 79 HNSCC patients(Peng et al. 2011), GSE13601 consisting of 37 HNSCC patients(Estilo et al. 2009), GSE65858 from 270 HNSCC patients(Wichmann et al. 2015), GSE136037 from 49 HNSCC patients(Alfieri et al. 2020), and The Cancer Genome Atlas (TCGA) -HNSC cohorts including 546 HNSCC HTSeq-counts, methylation profiles and related clinical information were downloaded. CalcNormFactors was used to calculate normalization factors to scale the gene expression in TCGA dataset (Anders \& Huber 2010; Le et al. 2019). Youden index (sensitivity + specificity -1 ) was used to calculate the best cutoff of survival analysis by R package ("SurvivalROC")(Huang et al. 2017; Luo \& Xiong 2013). GSE65858 and GSE136037 dataset from the GEO database were converted to transcripts per million (TPM)(Zhao et al. 2020a). And the term "N-" means primary lesions in HNSCC patients without neck lymph node metastasis, and "N+" means primary lesions in HNSCC patients with neck lymph node metastasis. The normalized data provided from original studies in other datasets were used in this study directly.

\section{Survival analysis}

Briefly, the expression of PLAU was categorized into low or high by 'Maximally Selected Rank Statistics' (maxstat) method(Lausen \& Schumacher 1992). The cutoff value of PLAU mRNA expression in TCGA was 9532 in Table 1. Older or younger is classified based on its mean age. The overall survival (OS), progression-free interval (PFI) or recurrence free survival (RSF) curves were visualized by Kaplan-Meier plots. Univariate and multivariate Cox regression analysis was applied to death hazard ratios calculation after the proportional hazard assumption was tested.

\section{Go analysis and co-expression network establishment}

Go enrichment analysis was performed by Bioconductor package "clusterProfiler" (Yu et al. 2012). The method of KEGG enrichment analysis was performed same as the Go enrichment analysis. The co-expression genes were screened using R packages ("limma").From the TCGAHNSCC databases, we used Pearson correlation coefficients (|Pearson correlation coefficient $\mid>$ 0.5 and P-value $<0.001$ ) and the z-test to examine the correlation between PLAU expression level and co-expression genes. STRING database and Cytoscape tool were used to construct the protein-protein-interactions (PPI) of genes (Struk et al. 2019).

\section{Immune cell environment analysis}

Estimation of stromal and immune cells in malignant tumor tissues using Expression data (ESTIMATE) is a tool for predicting tumor purity, and the presence of infiltrating stromal/immune cells in tumor tissues using gene expression data (Yoshihara et al. 2013). We used R packages 
118 ("estimate") to get immune-environment scores in HNSCC patients. R packages

119 (“CIBERSORT.R”, https://cibersortx.stanford.edu/ )(Newman et al. 2015) was used to 120 deconvoluted 22 common immune cell proportions in HNSCC patients. The correlation of PLAU 121 expression with immune environment and 22 immune cells were investigated by Pearson test.

122 Software and statistical analyses

123 GraphPad Prism 8 or R studio (version 3.5.3) was used to evaluate all data (Kamdem et al. 2019;

124 Le \& Huynh 2019). Chi-squre or Fisher's exact test was performed to compare the differences

125 expression of PLAU across different groups. $\mathrm{P}<0.05$ was considered statistically significant.

126 The detailed codes and other packages' information were included in the supplementary

127 materials.

\section{Results}

\section{PLAU mRNA is over-expressed in HNSCC}

To discover the expression of PLAU mRNA in HNSCC, we analyzed three independent patient cohorts, which showed a consistent result that PLAU mRNA expression was elevated in HNSCC tumors than normal tissues(all $\mathrm{p}<0.01$, Figure $1 \mathrm{~A}-\mathrm{C}$ ). And the overexpression of PLAU mRNA was confirmed in 10 different HNSCC cell lines as compared to 4 types of human keratinocyte cell lines ( $p=0.002$, Figure 1D). Furthermore, PLAU mRNA in HPV positive HNSCC samples was interposed between adjacent normal tissues and HPV negative tumors (Supplementary Figure 1A). There was significantly less PLAU mRNA in HPV positive tumors than HPV negative ones according to another patient cohort (Supplementary Figure 1B).

\section{Association of PLAU mRNA with neck lymph node status in HNSCC} In order to study the role of PLAU mRNA may play in HNSCC patients, the relationship of PLAU mRNA and clinical parameters was further characterized in TCGA-HNSC cohort. As shown in Table 2, there was no significantly different expression of PLAU mRNA in different age, gender, clinical stage, and tumor stage, but the difference of PLAU in HPV positivity and neck node status was considered as $\operatorname{significant}(\mathrm{p}=0.001, \mathrm{p}=0.033$, respectively). Higher expression of PLAU was founded in patients with neck lymph node metastasis than patient without neck lymph node metastasis in another independent cohort as well (Figure 1E).

\section{PLAU is an independent predictor of HNSCC prognosis}

Considering the above findings, we continued to analysis the possible correlation of HNSCC survival time and PLAU expression. As shown in Table 3, age, clinical stage, tumor size, neck lymph node status, HPV positivity and PLAU expression was considered to be significantly associated with overall survival time in univariate cox analysis of 496 HNSCC patients. Multivariate cox analysis indicated the hazard ratio of death was reached to 1.52 when high PLAU expressed HNSCC patients compared to patients with low PLAU expression after excluding the potential affections from age, tumor size, neck node metastasis and HPV positivity (Figure 2A, $\mathrm{p}=0.012$, 95\% CI: 1.09-2.10).

The survival curves of PLAU expression were visualized by Kaplan-Meier plots (Figure 2B), which implied that high PLAU expressed HNSCC patients had decreased overall survival probability than patients with low PLAU expression (Figure 2B). The same finding could be 
158 observed in another independent HNSCC cohort (Figure 2C). Additionally, high expression of

159 PLAU in HNSCC patients was founded to predict unfavorable outcomes in terms of PFI and

160 RSF (Figure 2D-E).

161 Performance of PLAU expression in predicting 5-year overall survival outcomes of 162 HNSCC patients

163 The predictive performance of PLAU expression in 5-year overall survival outcomes of HNSCC 164 patients was analyzed by ROC analysis. As shown in Figure 3, the areas under the ROC curve 165 (AUCs) of PLAU expression was 0.795, higher than HPV status(AUC: 0.531), neck node 166 status(AUC: 0.58) and tumor size stage(AUC: 0.532). Combining PLAU expression and neck 167 node status as an integrated factor (N+PLAU), the AUC was 0.862 , with an $88.5 \%$ sensitivity, $16864 \%$ specificity, $70.4 \%$ positive predictive value and $80.1 \%$ negative predictive value. Taken 169 together, PLAU itself or combined with neck node status may facilitate the overall survival 170 prediction for HNSCC patients.

\section{Hypomethylation of PLAU in HNSCC patients}

172 A growing body of literature reports that DNA methylation and mRNA expression most likely to

173

174

175

176

177

178

179

180

181

182

183

184

185

186

187

188

189

190

191

192

193

194

195

196

197

198

be negative correlated(Huang et al. 2015). Methylation profiles of 496 TCGA-HNSC patients were analyzed to investigate the possible upstream reasons for elevated PLAU mRNA expression in HNSCC tumors. As shown in Figure 4, the methylated level of PLAU was successively and significantly decreased in adjacent normal tissues, HPV positive tumors and HPV negative tumors (Figure 4A). The PLAU methylation and mRNA expression was negatively correlated (Figure 4B, $\mathrm{R}=-0.43, \mathrm{p}<0.001$ ). As expected, patients with hypomethylated PLAU, indicating higher expression of PLAU mRNA, had worse overall survival outcomes than patients with hypermethylated PLAU (Figure 4C).

\section{Network establishment for PLAU correlated genes in HNSCC}

To further understand the possible downstream reasons a total of 205 genes was correlated with PLAU expression in TCGA-HNSCC patients( 21 of 205 genes were negatively correlated with PLAU and 184 genes were positively correlated with PLAU). The top 20 genes of positively or negatively correlated with PLAU are shown in a Heatmap (Figure 5A). The interaction network of these 205 genes was established based on STRING and Cytoscape (Figure 5B). Next, we performed GO and KEGG enrichment analysis to understand the potential biological functions of PLAU in HNSCC. GO analysis showed that the major biological process (extracellular matrix organization), cellular component (extracellular matrix), and molecular functions (cell adhesion molecule binding) may contribute to PLAU related biology (Figure 5C). KEGG pathway analysis illuminated that the P13K-Akt signaling pathway, human papillomavirus infection, proteoglycans in cancer, and focal adhesion as significantly enriched by the PLAU co-expressed genes (Figure 5D).

\section{Distributions of tumor infiltrating immune cell in HNSCC patients with different PLAU expression}

More and more evidence revealed the tumor immune microenvironment is a crucial factor in tumor biology(Mao et al. 2020). To interpret the role of PLAU expression in HNSCC based on immunity conception, the scores or proportions of tumor infiltrating cells were compared in

Peer] reviewing PDF | (2020:07:50837:1:1:NEW 12 Dec 2020) 
199 TCGA-HNSCC cohort. It shows that PLAU expression was positively correlated with stromal

200

201

202

203

204

205

206

207

208

209

210

211

212

213

214

215

216

217

218

219

220

221

222

223

224

225

226

227

228

229

230

231

232

233

234

235

236

237

238

score (Figure 6A). Further analysis found the expression level of PLAU was positively correlated with M1 type macrophages, negatively associated with CD4 + T cell, Tregs cell, and follicular helper T cell (Figure 6B-E) (All p<0.05).

\section{Discussion}

Our study, using multiple publicly available profiles in HNSCC cohorts and cell lines, confirmed that PLAU mRNA was over-expressed and associated with neck node lymph metastasis in HNSCC tumors. And, we showed that PLAU expression might be an independent prognosis index for HNSCC patients, which consistent with many other cancer reports(Mahmood et al. 2018)including breast cancer, prostate cancer, ovarian cancer, sarcoma, melanoma, gastric cancer, esophageal cancer, and colorectal cancer. Furthermore, the DNA methylation level and mRNA expression level of PLAU was investigated in our work. As far as we know, this is the first report to link PLAU methylation level with its mRNA expression in cancer samples. There are many possible explanations, such as generic regulation, epigenetic modulation, or mRNA decay, for aberrant expression of mRNA like PLAU. Our work implied that the increased expression of PLAU in HNSCC tumors might be contributed by its hypomethylated levels to some extent. Genetic mutations and epigenetic alterations have critical functions in modulating oncogenes' transcription in human carcinomas(Wang et al. 2019b; Zhou et al. 2019). The methylation values of DNA could be a prognosis biomarker in cancer(Teixeira et al. 2019), which supported our finding that HNSCC patient with hypomethylated PLAU might have a worse survival outcome.

PLAU is a gene encodes for urokinase plasminogen activator (UPA). The detailed mechanism that underlying PLAU's role in HNSCC remain unclear. But it was indicated to involve in the transformation of inactive plasminogen into active plasminogen, which plays an important role in a series of transfer cascades(Choong \& Nadesapillai 2003). Previous research has shown that PLAU and type plasminogen activator (tPA) mediated the plasminogen activator (PA) system(Mahmood et al. 2018). PLAU can increase cell proliferation through the activate growth factors or adhesion molecules, for example, VEGF, TGF- $\beta$ and the $\alpha 5 \beta 1$ integrins(Aguirre-Ghiso et al. 2001; Duffy 2004; Ulisse et al. 2009). PLAU could increase cell adhesion and migration during metastasis and proliferation of tumor cells(Zhao et al. 2020b), which may explain our finding that elevated expression of PLAU in node metastasis tumors. Under hypoxia conditions, PLAU expression can activate downstream Akt and Rac1 signaling pathways, thus promoting EMT and cell invasion(Lester et al. 2007).In our GO and pathway enrichment assays, PI3K-Akt pathway was enriched by PLAU co-expressed genes. Akt activation maybe the downstream pathway of PLAU leading to HNSCC cell invasion and metastasis.

Growing evidence suggests that cells (such as macrophages, T cells, neutrophils, lymphoid cells and so on) in the immune microenvironment are related to tumor escape and progression(Hinshaw \& Shevde 2019). Kipp Weiskopf et al. found that CD47 engaged signalregulatory protein alpha, which acts as an inhibitory receptor on macrophages to promote immune evasion(Weiskopf et al. 2016). Recent data suggest that exposure to immune checkpoint

Peer) reviewing PDF | (2020:07:50837:1:1:NEW 12 Dec 2020) 
239

240

inhibitors (ICI) increase tumor sensitivity to chemotherapy in HNSCC(Saleh et al. 2019). Therefore, the investigation of the relationship between HNSCC and the immune microenvironment may help us to both diagnose and treat more effectively. In our study, we showed that PLAU expression is positively correlated with stromal score. The stromal-immune score represents a prognosis stratification tool intended to be developed as reliable prognostic signatures in gastric cancer (Wang et al. 2019a). Aberrance of macrophage function significantly contributes to disease progressions, such as in the case of cancer, fibrosis, and diabetes (Ngambenjawong et al. 2017). By analyzing the immune cell proportions, we identified PLAU expression was positively correlated with M1 type macrophages, and negative association with CD4+ T cell, Tregs cell, and follicular helper T cell. These associations could explain the role of PLAU in HNSCC prognosis from the immunological respective. In addition, we found that PLAU expression was reduced in HPV positive HNSCC tumors as compared to HPV negative ones. HPV positivity is well-accepted as a strong survival favorable factor in head and neck cancer patients, which indirectly supports that low expression of PLAU predicts a better survival in HNSCC patients. And PLAU activity could be a partial reason for HPV's role in HNSCC tumors.

Back to clinical significance, the performances of PLAU and other independent prognosis indicators in predicting HNSCC 5-year survival outcome were investigated. Although HPV status, tumor size or neck node status is independent prognosis indicator in HNSCC, their AUC is very low according to the ROC assays. However, the AUC of PLAU expression reach 0.795, higher than other clinical parameters. Moreover, the combination of PLAU and neck node status could predict HNSCC 5-year overall survival outcomes with an $88.5 \%$ sensitivity and $64 \%$ specificity, which demonstrated the capability of PLAU expression in HNSCC prognosis. Certainly, we need to be aware that our findings require the further validations from in vivo and vitro experiments although the conclusion was confirmed across five independent cohorts. Another limitation in this study is the platform used in different cohorts is different, which may produce bias to the data analysis and bring hardness for the deep integrated analysis.

\section{Conclusions}

All in all, PLAU might be an independent biomarker for predicting outcomes of HNSCC patients. The elevated expression of PLAU was associated with HPV positivity and neck node status. PI3K-Akt pathway and aberrant proportions of immune cells might underly the mechanism of PLAU's oncogene role in HNSCC.

\section{Acknowledgements}

This research was supported by the Project of Hunan Health Commission (B2019165), National Natural Science Foundation of China (Nos. 81974424, 81874133, 81772903, and 81602389), Natural Science Foundation of Hunan Province (Nos. 2020JJ4827, 2019JJ50944, and 2018JJ2630) and the Huxiang Young Talent Project (No. 2018RS3024).

\section{References}


Aguirre-Ghiso JA, Liu D, Mignatti A, Kovalski K, and Ossowski L. 2001. Urokinase receptor and fibronectin regulate the ERK(MAPK) to p38(MAPK) activity ratios that determine carcinoma cell proliferation or dormancy in vivo. Molecular biology of the cell 12:863-879.

Ai C, Zhang J, Lian S, Ma J, Győrffy B, Qian Z, Han Y, and Feng Q. 2020. FOXM1 functions collaboratively with PLAU to promote gastric cancer progression. Journal of Cancer 11:788-794. 10.7150/jca.37323

Alfieri S, Carenzo A, Platini F, Serafini MS, Perrone F, Galbiati D, Sponghini AP, Depenni R, Vingiani A, Quattrone P, Marchesi E, Iannó MF, Micali A, Mancinelli E, Orlandi E, Marceglia S, Locati LD, Licitra L, Bossi P, and De Cecco L. 2020. Tumor Biomarkers for the Prediction of Distant Metastasis in Head and Neck Squamous Cell Carcinoma. Cancers 12. 10.3390/cancers12040922

Anders S, and Huber W. 2010. Differential expression analysis for sequence count data. Genome biology 11:R106. 10.1186/gb-2010-11-10-r106

Chen H-H, Yu H-I, Yang M-H, and Tarn W-Y. 2018. DDX3 Activates CBC-eIF3-Mediated Translation of uORFContaining Oncogenic mRNAs to Promote Metastasis in HNSCC. Cancer research 78:4512-4523. 10.1158/0008-5472.CAN-18-0282

Choong PFM, and Nadesapillai APW. 2003. Urokinase plasminogen activator system: a multifunctional role in tumor progression and metastasis. Clinical orthopaedics and related research:S46-S58.

Cramer JD, Burtness B, Le QT, and Ferris RL. 2019. The changing therapeutic landscape of head and neck cancer. Nature reviews Clinical oncology 16:669-683. 10.1038/s41571-019-0227-z

Danø K, Behrendt N, Høyer-Hansen G, Johnsen M, Lund LR, Ploug M, and Rømer J. 2005. Plasminogen activation and cancer. Thrombosis and haemostasis 93:676-681.

Duffy MJ. 2004. The urokinase plasminogen activator system: role in malignancy. Current pharmaceutical design 10:39-49.

Estilo CL, O-charoenrat P, Talbot S, Socci ND, Carlson DL, Ghossein R, Williams T, Yonekawa Y, Ramanathan Y, Boyle JO, Kraus DH, Patel S, Shaha AR, Wong RJ, Huryn JM, Shah JP, and Singh B. 2009. Oral tongue cancer gene expression profiling: Identification of novel potential prognosticators by oligonucleotide microarray analysis. BMC cancer 9:11. 10.1186/1471-2407-9-11

Gutierrez LS, Schulman A, Brito-Robinson T, Noria F, Ploplis VA, and Castellino FJ. 2000. Tumor development is retarded in mice lacking the gene for urokinase-type plasminogen activator or its inhibitor, plasminogen activator inhibitor-1. Cancer research 60:5839-5847.

Hanahan D, and Weinberg RA. 2011. Hallmarks of cancer: the next generation. Cell 144:646-674. 10.1016/j.cell.2011.02.013

Hinshaw DC, and Shevde LA. 2019. The Tumor Microenvironment Innately Modulates Cancer Progression. Cancer research 79:4557-4566. 10.1158/0008-5472.CAN-18-3962

Huang R, Liao X, and Li Q. 2017. Identification and validation of potential prognostic gene biomarkers for predicting survival in patients with acute myeloid leukemia. OncoTargets and therapy 10:5243-5254. 10.2147/OTT.S147717

Huang W-Y, Hsu S-D, Huang H-Y, Sun Y-M, Chou C-H, Weng S-L, and Huang H-D. 2015. MethHC: a database of DNA methylation and gene expression in human cancer. Nucleic acids research 43:D856-D861. 10.1093/nar/gku1151

Kamdem SD, Konhawa F, Kuemkon EM, Meyo Kamguia L, Tchanana GK, Nche F, Oumarou A, Hamza M, Ouratou Y, Tcheutchoua MN, Ghislain Essomba R, Ngogang MP, Kengne M, Netongo PM, Ondigui BE, 
319

320

321

322

323

324

325

326

327

328

329

330

331

332

333

334

335

336

337

338

339

340

341

342

343

344

345

346

347

348

349

350

351

352

353

354

355

356

357

358

359

Okomo Assoumou MC, Brombacher F, and Nono JK. 2019. Negative Association of Interleukin-33 Plasma Levels and Schistosomiasis Infection in a Site of Polyparasitism in Rural Cameroon. Frontiers in immunology 10:2827. 10.3389/fimmu.2019.02827

Kang H, Kiess A, and Chung CH. 2015. Emerging biomarkers in head and neck cancer in the era of genomics. Nature reviews Clinical oncology 12:11-26. 10.1038/nrclinonc.2014.192

Kowalski LP, Carvalho AL, Martins Priante AV, and Magrin J. 2005. Predictive factors for distant metastasis from oral and oropharyngeal squamous cell carcinoma. Oral oncology 41:534-541.

Lausen B, and Schumacher M. 1992. Maximally Selected Rank Statistics. Biometrics 48:73-85. 10.2307/2532740

Le NQK, and Huynh T-T. 2019. Identifying SNAREs by Incorporating Deep Learning Architecture and Amino Acid Embedding Representation. Frontiers in physiology 10:1501. 10.3389/fphys.2019.01501

Le NQK, Huynh T-T, Yapp EKY, and Yeh H-Y. 2019. Identification of clathrin proteins by incorporating hyperparameter optimization in deep learning and PSSM profiles. Computer methods and programs in biomedicine 177:81-88. 10.1016/j.cmpb.2019.05.016

Leemans CR, Snijders PJF, and Brakenhoff RH. 2018. The molecular landscape of head and neck cancer. Nature reviews Cancer 18:269-282. 10.1038/nrc.2018.11

Lester RD, Jo M, Montel V, Takimoto S, and Gonias SL. 2007. UPAR induces epithelial-mesenchymal transition in hypoxic breast cancer cells. The Journal of cell biology 178:425-436.

Li Y, Lu Z, Che Y, Wang J, Sun S, Huang J, Mao S, Lei Y, Chen Z, and He J. 2017. Immune signature profiling identified predictive and prognostic factors for esophageal squamous cell carcinoma. Oncoimmunology 6:e1356147. 10.1080/2162402X.2017.1356147

Lin M, Zhang Z, Gao M, Yu H, Sheng H, and Huang J. 2019. MicroRNA-193a-3p suppresses the colorectal cancer cell proliferation and progression through downregulating the PLAU expression. Cancer management and research 11:5353-5363. 10.2147/CMAR.S208233

Luo J, and Xiong C. 2013. Youden index and Associated Cut-points for Three Ordinal Diagnostic Groups. Communications in statistics: Simulation and computation 42:1213-1234.

Mahmood N, Mihalcioiu C, and Rabbani SA. 2018. Multifaceted Role of the Urokinase-Type Plasminogen Activator (uPA) and Its Receptor (uPAR): Diagnostic, Prognostic, and Therapeutic Applications. Frontiers in oncology 8:24. 10.3389/fonc.2018.00024

Mao M, Yu Q, Huang R, Lu Y, Wang Z, and Liao L. 2020. Stromal score as a prognostic factor in primary gastric cancer and close association with tumor immune microenvironment. Cancer medicine. 10.1002/cam4.2801

Moertel CG, Fleming TR, Macdonald JS, Haller DG, Laurie JA, Tangen CM, Ungerleider JS, Emerson WA, Tormey DC, Glick JH, Veeder MH, and Mailliard JA. 1995. Fluorouracil plus levamisole as effective adjuvant therapy after resection of stage III colon carcinoma: a final report. Annals of internal medicine 122:321-326.

Murphy DA, and Courtneidge SA. 2011. The 'ins' and 'outs' of podosomes and invadopodia: characteristics, formation and function. Nature reviews Molecular cell biology 12:413-426. 10.1038/nrm3141

Newman AM, Liu CL, Green MR, Gentles AJ, Feng W, Xu Y, Hoang CD, Diehn M, and Alizadeh AA. 2015. Robust enumeration of cell subsets from tissue expression profiles. Nature methods 12:453-457. 10.1038/nmeth. 3337

Ngambenjawong C, Gustafson HH, and Pun SH. 2017. Progress in tumor-associated macrophage (TAM)-targeted therapeutics. Advanced drug delivery reviews 114:206-221. 10.1016/j.addr.2017.04.010 
Novak CM, Horst EN, Taylor CC, Liu CZ, and Mehta G. 2019. Fluid shear stress stimulates breast cancer cells to display invasive and chemoresistant phenotypes while upregulating PLAU in a 3D bioreactor. Biotechnology and bioengineering 116:3084-3097. 10.1002/bit.27119

Peng C-H, Liao C-T, Peng S-C, Chen Y-J, Cheng A-J, Juang J-L, Tsai C-Y, Chen T-C, Chuang Y-J, Tang C-Y, Hsieh W-P, and Yen T-C. 2011. A novel molecular signature identified by systems genetics approach predicts prognosis in oral squamous cell carcinoma. PloS one 6:e23452. 10.1371/journal.pone.0023452

Saleh K, Daste A, Martin N, Pons-Tostivint E, Auperin A, Herrera-Gomez RG, Baste-Rotllan N, Bidault F, Guigay J, Le Tourneau C, Saada-Bouzid E, and Even C. 2019. Response to salvage chemotherapy after progression on immune checkpoint inhibitors in patients with recurrent and/or metastatic squamous cell carcinoma of the head and neck. European journal of cancer (Oxford, England : 1990) 121:123-129. 10.1016/j.ejca.2019.08.026

Siu LL, Even C, Mesía R, Remenar E, Daste A, Delord J-P, Krauss J, Saba NF, Nabell L, Ready NE, Braña I, Kotecki N, Zandberg DP, Gilbert J, Mehanna H, Bonomi M, Jarkowski A, Melillo G, Armstrong JM, Wildsmith S, and Fayette J. 2019. Safety and Efficacy of Durvalumab With or Without Tremelimumab in Patients With PD-L1-Low/Negative Recurrent or Metastatic HNSCC: The Phase 2 CONDOR Randomized Clinical Trial. JAMA oncology 5:195-203. 10.1001/jamaoncol.2018.4628

Struk S, Jacobs A, Sánchez Martín-Fontecha E, Gevaert K, Cubas P, and Goormachtig S. 2019. Exploring the protein-protein interaction landscape in plants. Plant, cell \& environment 42:387-409. 10.1111/pce.13433

Teixeira VH, Pipinikas CP, Pennycuick A, Lee-Six H, Chandrasekharan D, Beane J, Morris TJ, Karpathakis A, Feber A, Breeze CE, Ntolios P, Hynds RE, Falzon M, Capitanio A, Carroll B, Durrenberger PF, Hardavella G, Brown JM, Lynch AG, Farmery H, Paul DS, Chambers RC, McGranahan N, Navani N, Thakrar RM, Swanton C, Beck S, George PJ, Spira A, Campbell PJ, Thirlwell C, and Janes SM. 2019. Deciphering the genomic, epigenomic, and transcriptomic landscapes of pre-invasive lung cancer lesions. Nature medicine 25:517-525. 10.1038/s41591-018-0323-0

Torre LA, Bray F, Siegel RL, Ferlay J, Lortet-Tieulent J, and Jemal A. 2015. Global cancer statistics, 2012. CA: $a$ cancer journal for clinicians 65.10 .3322 /caac. 21262

Ulisse S, Baldini E, Sorrenti S, and D'Armiento M. 2009. The urokinase plasminogen activator system: a target for anti-cancer therapy. Current cancer drug targets 9:32-71.

Wang H, Wu X, and Chen Y. 2019a. Stromal-Immune Score-Based Gene Signature: A Prognosis Stratification Tool in Gastric Cancer. Frontiers in oncology 9:1212. 10.3389/fonc.2019.01212

Wang X, Xu Z, Ren X, Chen X, Wei J, Lin W, Li Z, Ou C, Gong Z, and Yan Y. 2019b. Function of low ADARB1 expression in lung adenocarcinoma. PloS one 14:e0222298. 10.1371/journal.pone.0222298

Weiskopf K, Jahchan NS, Schnorr PJ, Cristea S, Ring AM, Maute RL, Volkmer AK, Volkmer J-P, Liu J, Lim JS, Yang D, Seitz G, Nguyen T, Wu D, Jude K, Guerston H, Barkal A, Trapani F, George J, Poirier JT, Gardner EE, Miles LA, de Stanchina E, Lofgren SM, Vogel H, Winslow MM, Dive C, Thomas RK, Rudin CM, van de Rijn M, Majeti R, Garcia KC, Weissman IL, and Sage J. 2016. CD47-blocking immunotherapies stimulate macrophage-mediated destruction of small-cell lung cancer. The Journal of clinical investigation 126:2610-2620. 10.1172/JCI81603

Wichmann G, Rosolowski M, Krohn K, Kreuz M, Boehm A, Reiche A, Scharrer U, Halama D, Bertolini J, Bauer U, Holzinger D, Pawlita M, Hess J, Engel C, Hasenclever D, Scholz M, Ahnert P, Kirsten H, Hemprich A, Wittekind C, Herbarth O, Horn F, Dietz A, and Loeffler M. 2015. The role of HPV RNA transcription, 
401

402

403

404

405

406

407

408

409

410

411

412

413

414

415

416

417

418

419

420

421

422

423

424

425

426

427

428

\section{1}

26

27

immune response-related gene expression and disruptive TP53 mutations in diagnostic and prognostic profiling of head and neck cancer. International journal of cancer 137:2846-2857. 10.1002/ijc.29649

Yang K, Zhang S, Zhang D, Tao Q, Zhang T, Liu G, Liu X, and Zhao T. 2019. Identification of SERPINE1, PLAU and ACTA1 as biomarkers of head and neck squamous cell carcinoma based on integrated bioinformatics analysis. International journal of clinical oncology 24:1030-1041. 10.1007/s10147-019-01435-9

Yoshihara K, Shahmoradgoli M, Martínez E, Vegesna R, Kim H, Torres-Garcia W, Treviño V, Shen H, Laird PW, Levine DA, Carter SL, Getz G, Stemke-Hale K, Mills GB, and Verhaak RGW. 2013. Inferring tumour purity and stromal and immune cell admixture from expression data. Nature communications 4:2612. $10.1038 /$ ncomms 3612

Yu G, Wang L-G, Han Y, and He Q-Y. 2012. clusterProfiler: an R package for comparing biological themes among gene clusters. Omics : a journal of integrative biology 16:284-287. 10.1089/omi.2011.0118

Zhao S, Ye Z, and Stanton R. 2020a. Misuse of RPKM or TPM normalization when comparing across samples and sequencing protocols. RNA (New York, NY) 26:903-909. 10.1261/rna.074922.120

Zhao X, Liu Z, Ren Z, Wang H, Wang Z, Zhai J, Cao D, Lyu S, Li L, Lang R, and He Q. 2020b. Triptolide inhibits pancreatic cancer cell proliferation and migration via down-regulating PLAU based on network pharmacology of Tripterygium wilfordii Hook F. European journal of pharmacology 880:173225. 10.1016/j.ejphar.2020.173225

Zhou S, Yan Y, Chen X, Wang X, Zeng S, Qian L, Wei J, Yang X, Zhou Y, Gong Z, and Xu Z. 2019. Roles of highly expressed PAICS in lung adenocarcinoma. Gene 692:1-8. 10.1016/j.gene.2018.12.064

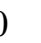


Figure 1

PLAU MRNA is over-expressed in HNSCC

(A, B) The expression of PLAU mRNA in normal and HNSCC tumor tissues was detected from the GEO databases. (C) Compared the difference expression of PLAU mRNA between tumor tissues and pair normal tissues in HNSCC. (D) The overexpression of PLAU mRNA was confirmed in HNSCC cell lines and human keratinocyte cell lines. (E) The difference expression of PLAU mRNA in neck lymph node status. "N-" means patients without neck lymph node metastasis, and "N+" means patients with neck lymph node metastasis. 
A

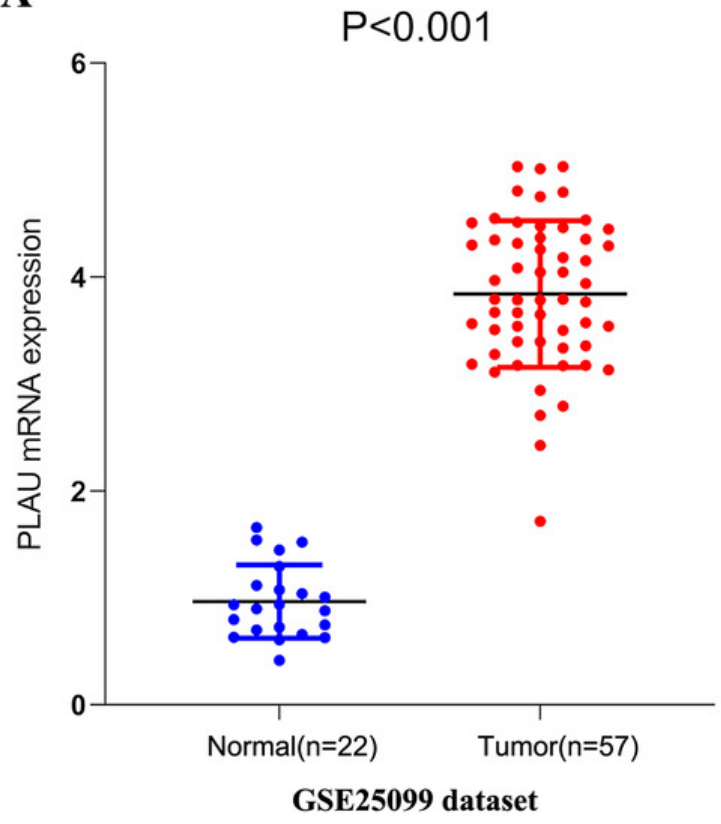

C

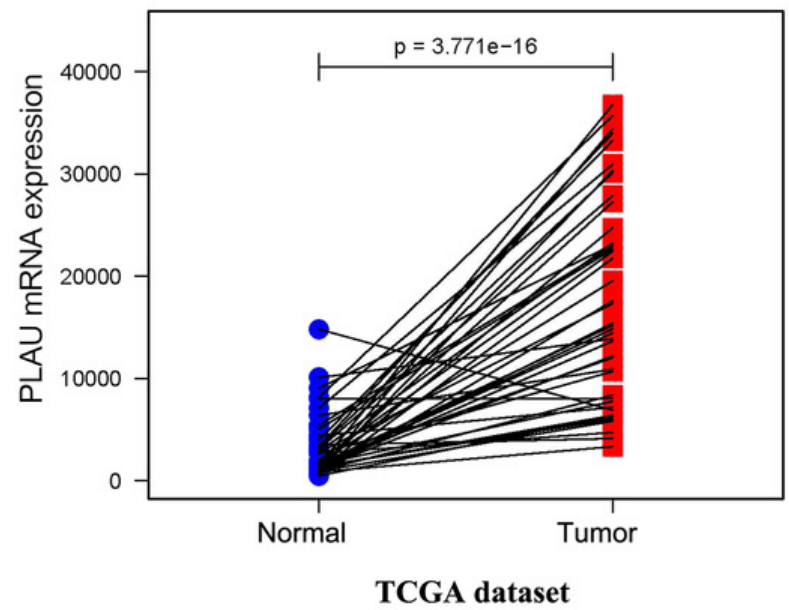

B

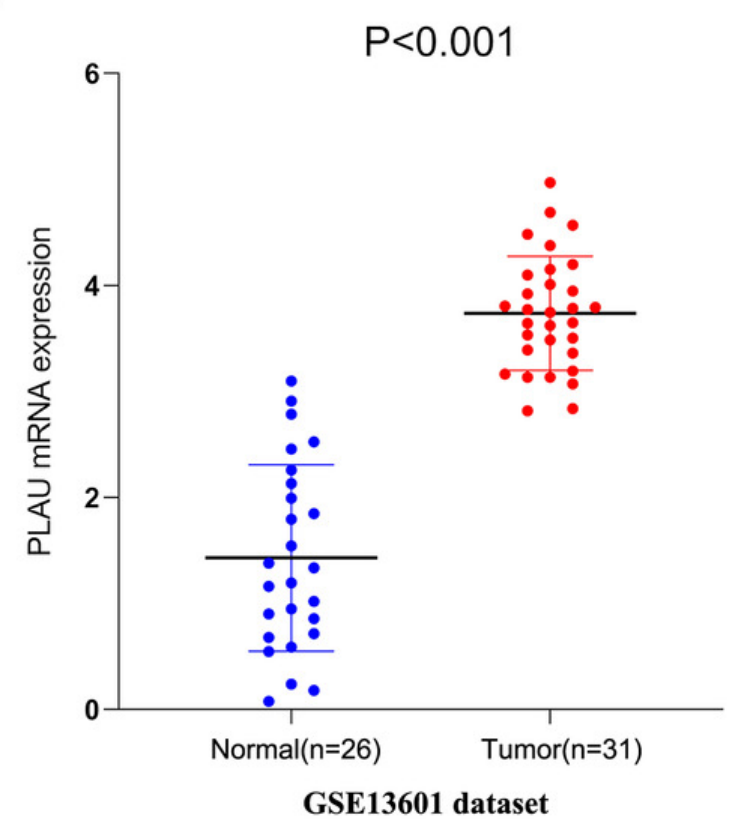

D

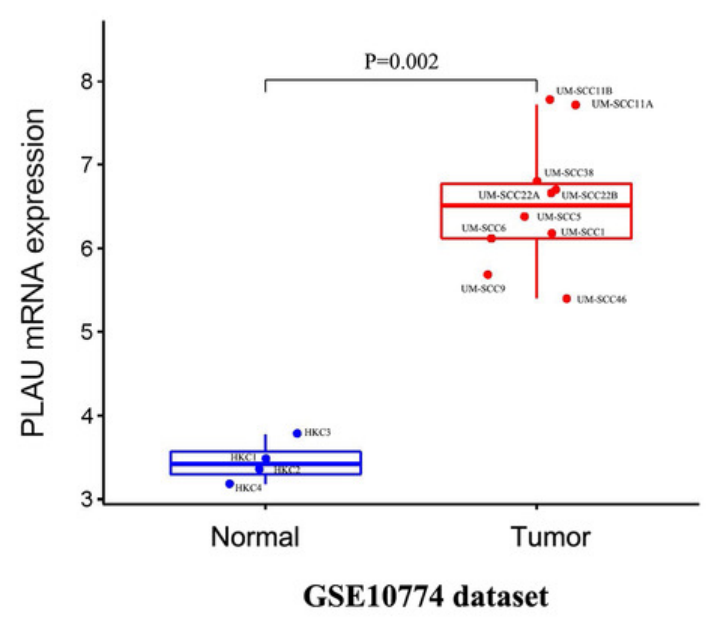

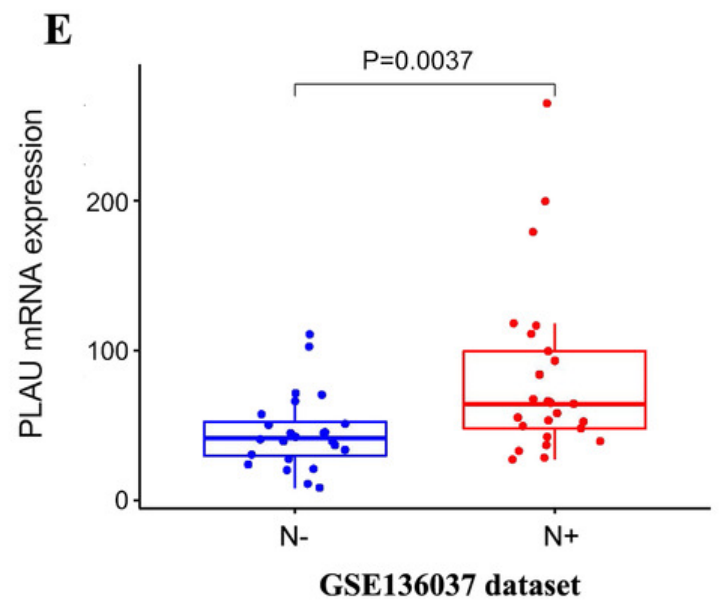


Figure 2

PLAU is an independent predictor of HNSCC prognosis

(A) Multivariate cox analysis related to PLAU. (B, C) The TCGA dataset and the GSE65858 database were used to assess the effect of PLAU expression on overall survival (OS). (D,E) The TCGA dataset assessed the effect of PLAU expression on progression-free interval (PFI) and relapse free survival (RFS) 
A

Multivariate cox analysis

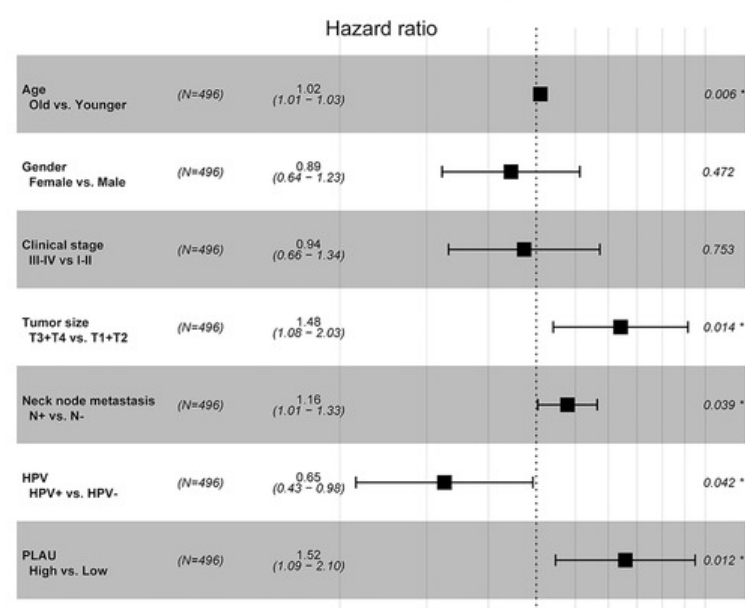

$0.4 \quad 0.6 \quad 0.8 \quad 1.21 .41 .61 .8222$

C

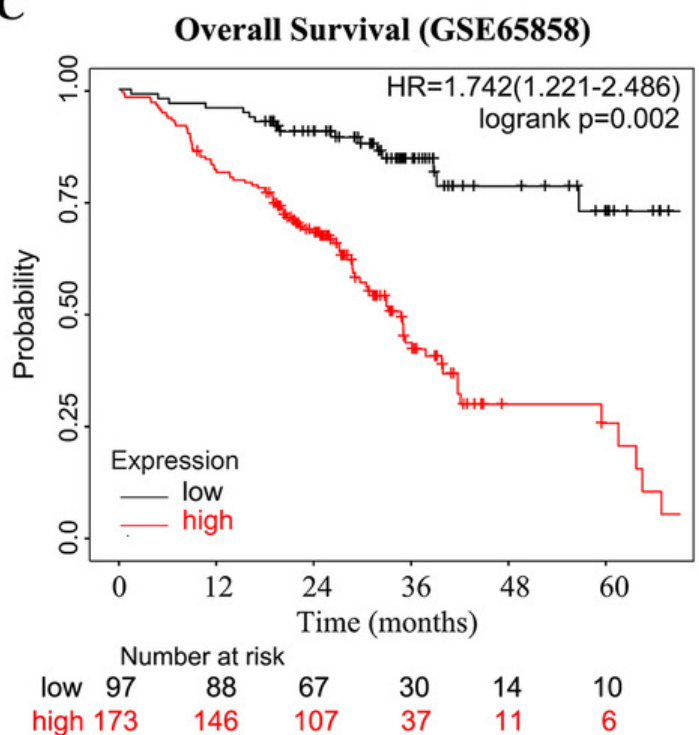

$\mathbf{E}$

E Recurrence Free Survival(TCGA)

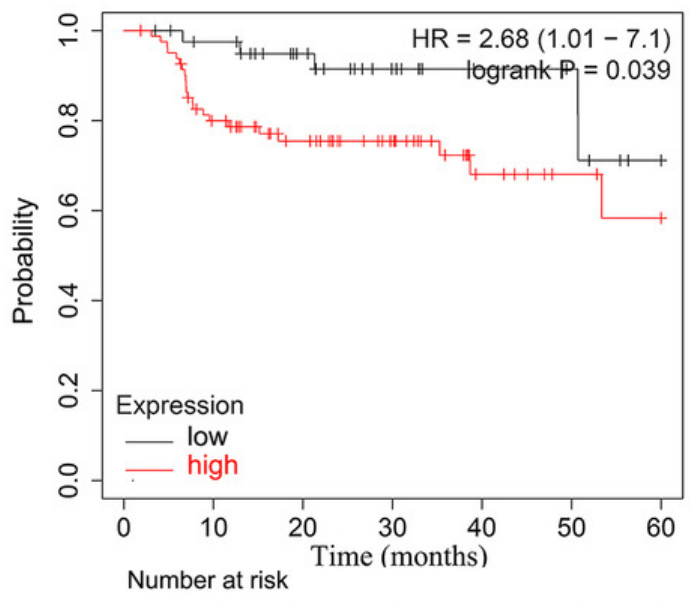

$\begin{array}{lllllll}\text { low } 44 & 40 & 24 & 12 & 11 & 4 & 2\end{array}$

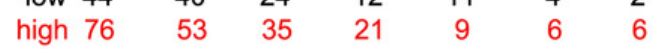

B

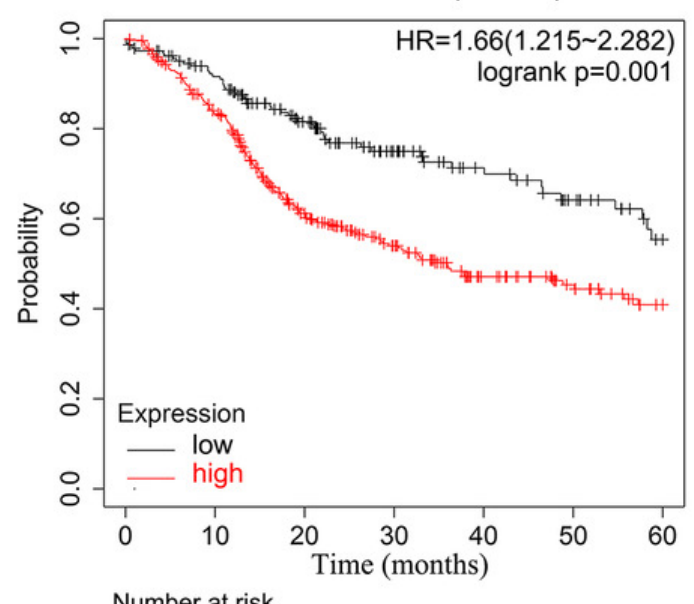

$\begin{array}{lllllll}\text { low } 184 & 141 & 80 & 48 & 31 & 17 & 11\end{array}$

D

high $312 \quad 215 \quad 112 \quad 72 \quad 42 \quad 23 \quad 18$

Progression-Free Interval(TCGA)

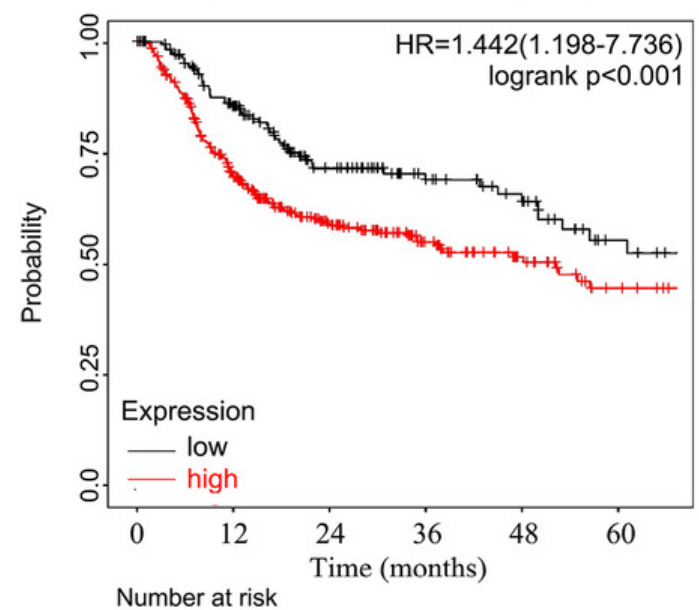

$\begin{array}{llllll}\text { low } 184 & 133 & 79 & 51 & 37 & 19\end{array}$

high $\begin{array}{llllll}312 & 193 & 114 & 72 & 45 & 25\end{array}$


Figure 3

ROC analysis of the PLAU expression with the clinical parameters of HNSCC in TCGA datasets.

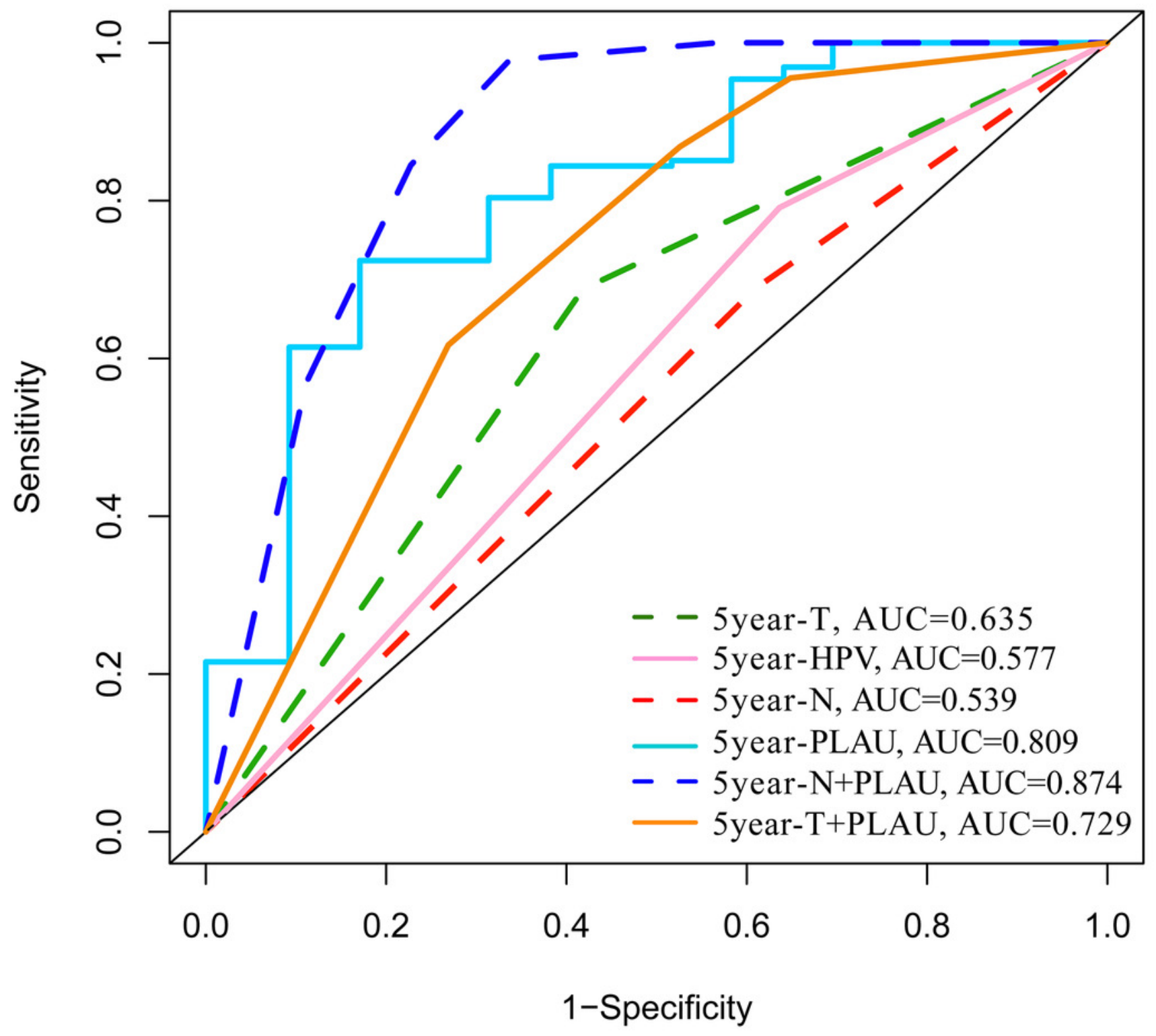


Figure 4

Relationship between PLAU methylation and HNSCC patients

(A) S eparating the groups by HPV status, PLAU methylation compared with the normal samples were evaluated in HNSCC samples of TCGA databases. (B) The correlation between the expression of PLAU and the beta value of methylation were evaluated by TCGA datasets. (C) The methylation of PLAU on OS by TCGA datasets.

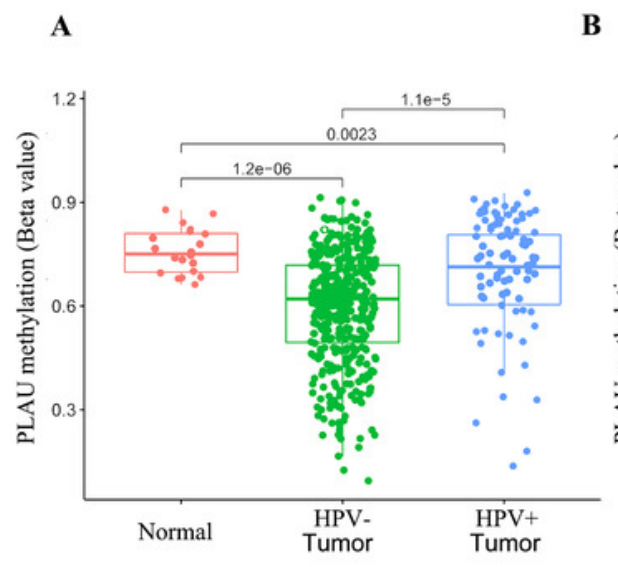

TCGA dataset
B

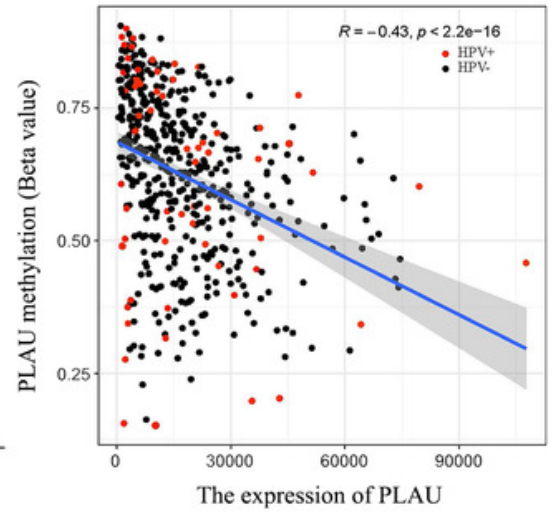

TCGA dataset
C

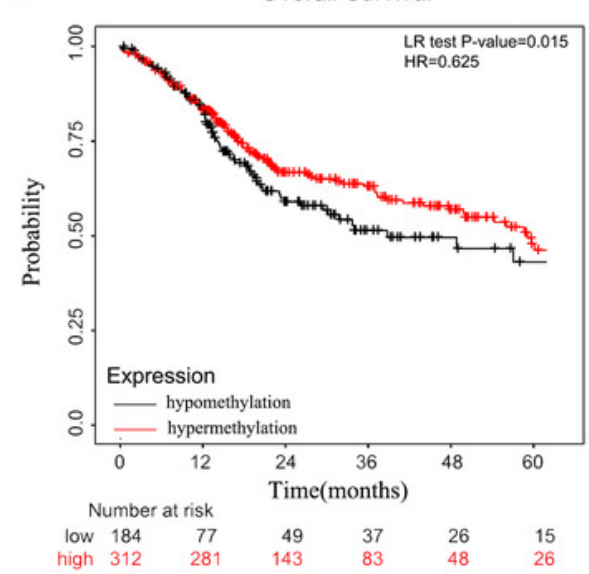

TCGA dataset 


\section{Figure 5}

Network establishment for PLAU correlated genes in HNSCC

(A) The top 20 genes of positively or negatively correlated with PLAU were showed in Heatmap. (B) The PPI networks of PLAU interaction partners generated by STRING and Cytoscape. The color represents the degree score (represent the intensity of the hub interacting with its neighbors). Degree score $<0.5$ represented low value (colored yellow), degree score $\geq 0.5$ represented high value (colored orange or red). (C) Major biological process, cellular component and molecular functions of PLAU biology by GO enrichment analysis. (D) KEGG pathway analyses further illuminate enriched function pathway related to PLAU. 
$\mathbf{A}$
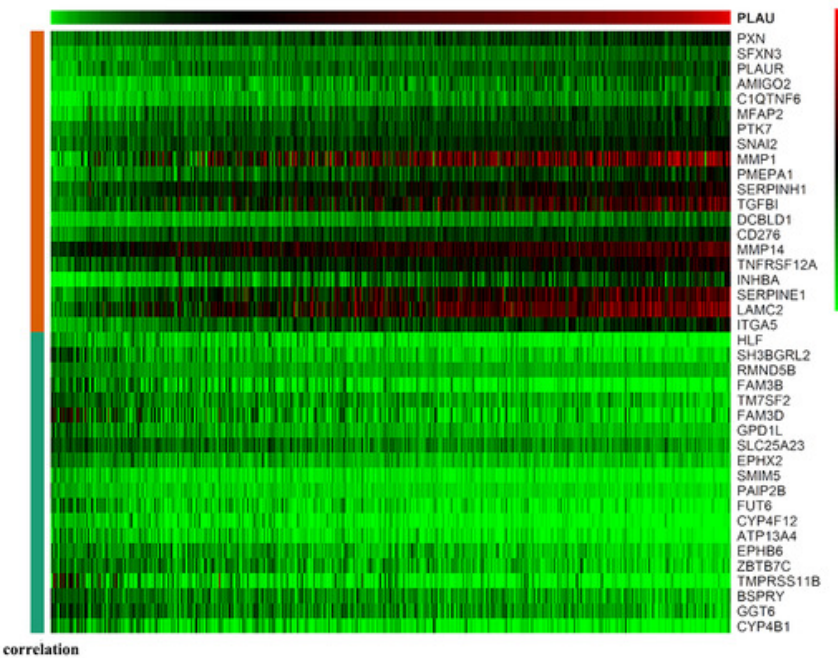

degree score

\section{B}

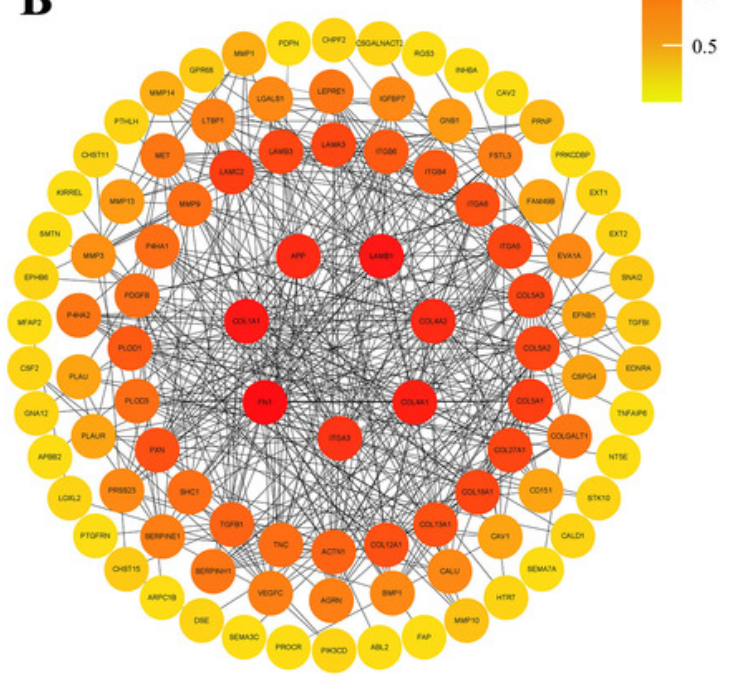

C

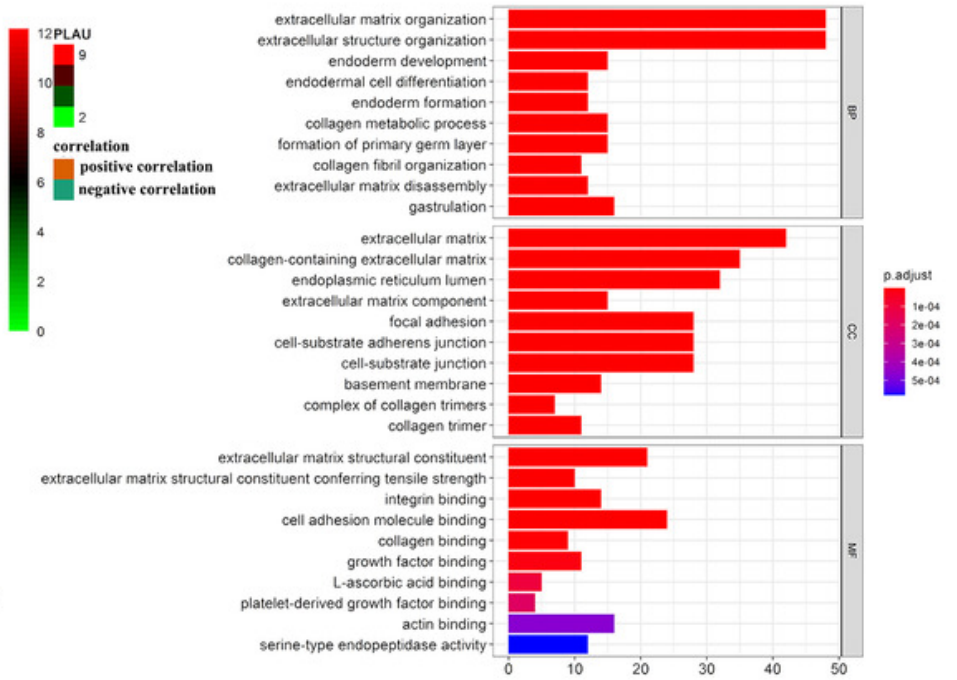

D

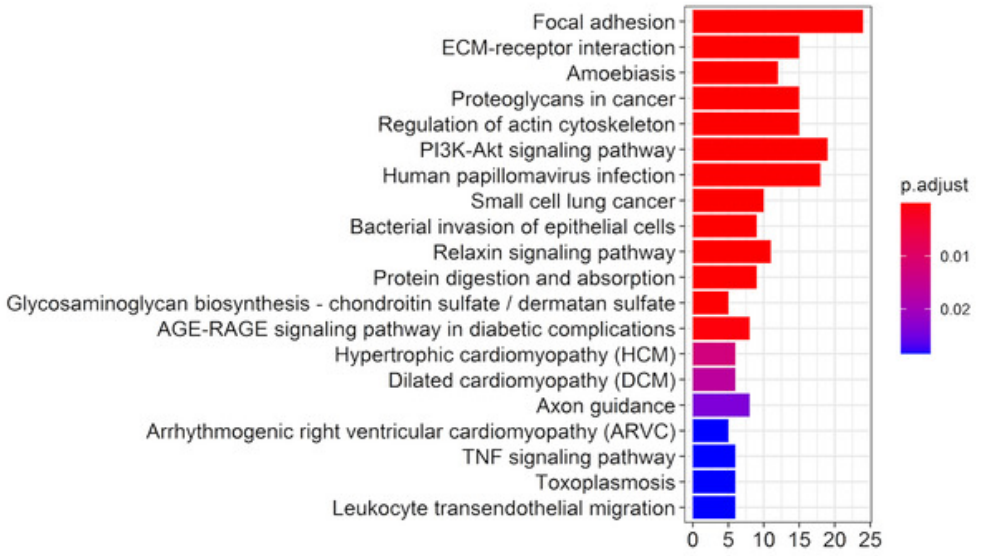


Figure 6

Relationship between PLAU and tumor immune microenvironment of HNSCC

(A) The expression of PLAU is positively correlated with stromal score. (B-E) The expression of PLAU was correlated with Tregs cell, M1 type macrophages, follicular helper T cell and CD4 $+\mathrm{T}$ cell. 
A

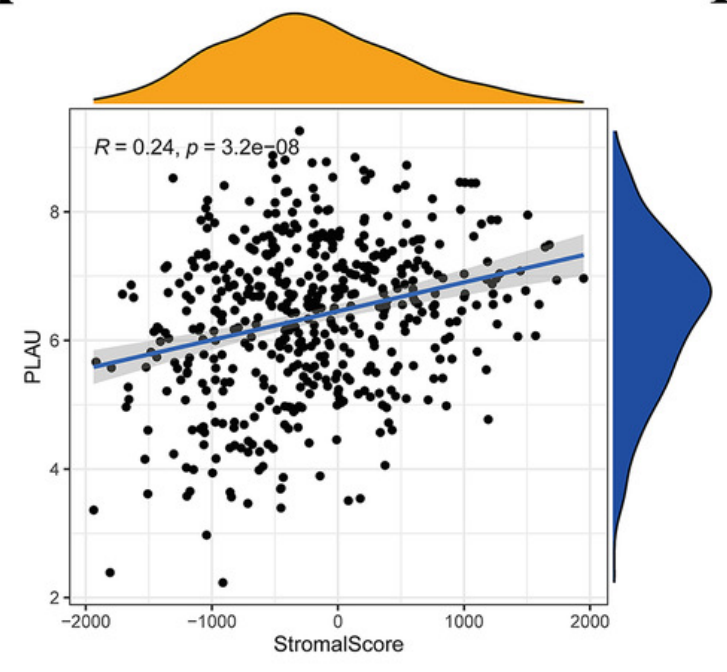

C

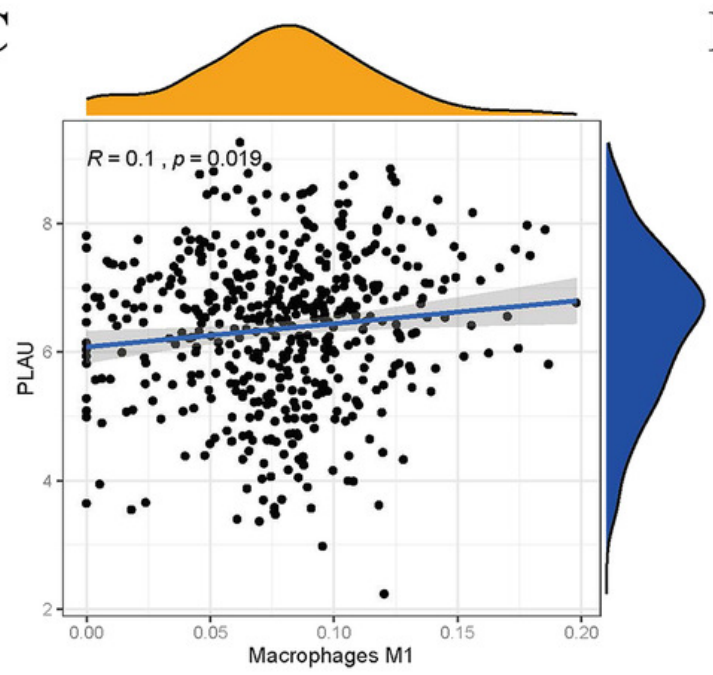

$\mathbf{E}$

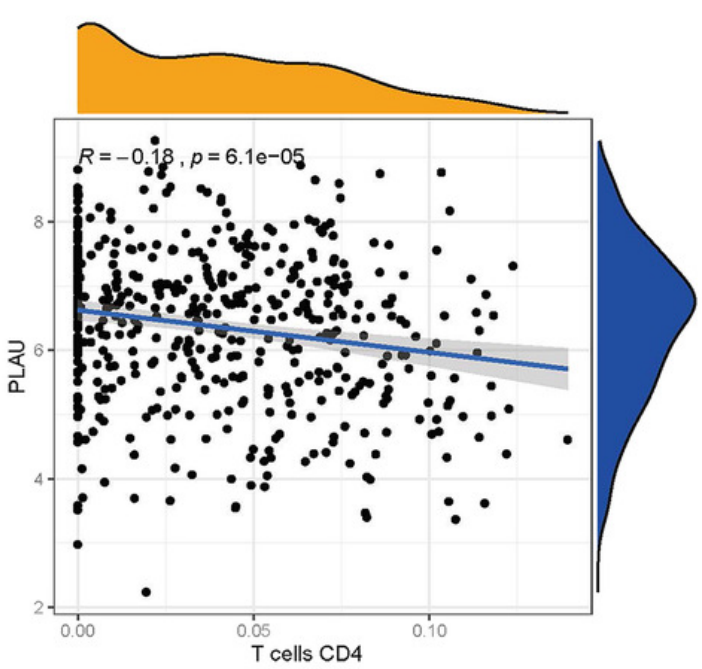

B

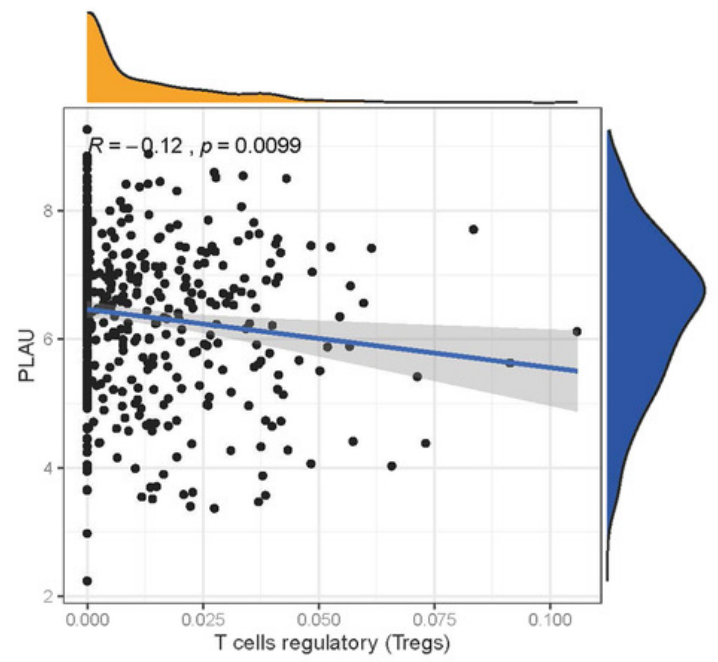

D

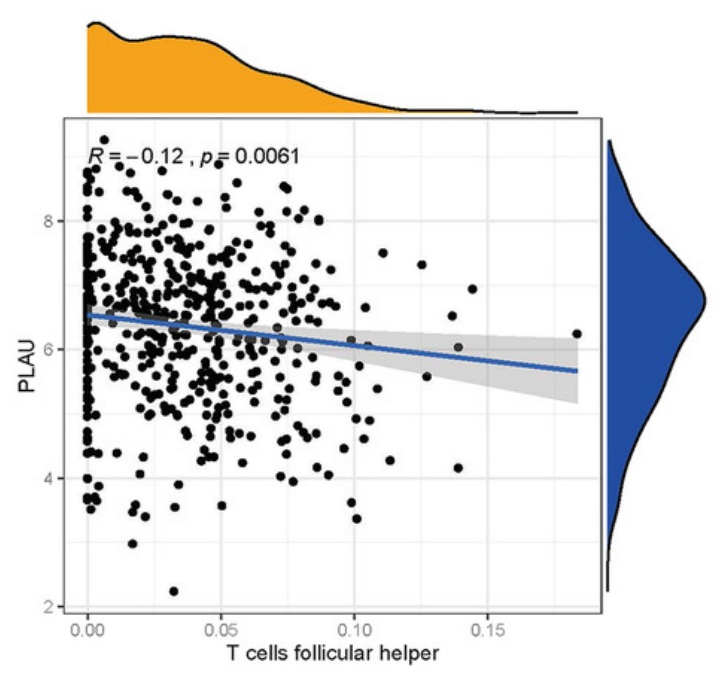


Table $\mathbf{1}$ (on next page)

Cutoff identification for survival time by clinical parameters 
1 Table 1. Cutoff identification for survival time by clinical parameters

\begin{tabular}{l|ccccccc}
\hline Index & Cutoff & AUC & $\mathbf{9 5 \% C I}$ & Sensitivity & Specificity & PPV & NPV \\
\hline $\mathbf{T}$ & $/$ & 0.532 & $0.451 \sim 0.616$ & 0.711 & 0.474 & 0.523 & 0.669 \\
$\mathbf{N}$ & $/$ & 0.58 & $0.495 \sim 0.668$ & 0.768 & 0.437 & 0.549 & 0.679 \\
HPV & $/$ & 0.531 & $0.437 \sim 0.624$ & 0.702 & 0.458 & 0.569 & 0.602 \\
PLAU & 9532 & 0.795 & $0.724 \sim 0.863$ & 0.901 & 0.679 & 0.749 & 0.798 \\
N+PLAU & $/$ & 0.862 & $0.663 \sim 0.887$ & 0.885 & 0.639 & 0.704 & 0.801 \\
T+PLAU & $/$ & 0.778 & $0.821 \sim 0.906$ & 0.995 & 0.619 & 0.748 & 0.812 \\
\hline
\end{tabular}

PPV: Positive Predictive Value. NPV: Negative Predictive Value 
Table 2 (on next page)

The PLAU expression in HNSCC patients with different clinical parameters 
1 Table 2. The PLAU expression in HNSCC patients with different clinical parameters

\begin{tabular}{|c|c|c|c|}
\hline \multirow[b]{2}{*}{ Clinical parameters } & \multicolumn{2}{|c|}{ PLAU mRNA expression } & \multirow[b]{2}{*}{ P-value } \\
\hline & $\begin{array}{c}\text { Low } \\
(n=184)\end{array}$ & $\begin{array}{c}\text { High } \\
(n=312)\end{array}$ & \\
\hline Age(years) & & & 0.949 \\
\hline Mean (SD) & $61.6(11.8)$ & $60.7(12.0)$ & \\
\hline Median [Min, Max] & $61.0[26.0,87.0]$ & $60.5[19,90]$ & \\
\hline Gender & & & 0.943 \\
\hline Female & $49(36.8 \%)$ & $84(37.2 \%)$ & \\
\hline Male & $135(63.2 \%)$ & $228(62.8 \%)$ & \\
\hline Clinical stage & & & 0.913 \\
\hline I-II & $136(37 \%)$ & $232(37.5 \%)$ & \\
\hline III-IV & $48(63 \%)$ & $80(62.5 \%)$ & \\
\hline Tumor stage & & & 0.23 \\
\hline $\mathrm{T} 1-2$ & $88(47.8 \%)$ & $126(40.4 \%)$ & \\
\hline T3-4 & $93(50.6 \%)$ & $182(58.3 \%)$ & \\
\hline Missing & $3(1.6 \%)$ & $4(1.3 \%)$ & \\
\hline Neck nodal metastasis & & & $0.033^{*}$ \\
\hline $\mathrm{N}-$ & $82(44.6 \%)$ & $105(33.7 \%)$ & \\
\hline $\mathrm{N}+$ & $101(54.9 \%)$ & $202(64.7 \%)$ & \\
\hline Missing & $1(0.5 \%)$ & $5(1.6 \%)$ & \\
\hline HPV & & & $0.001 * * *$ \\
\hline HPV- & $134(72.8 \%)$ & $270(86.5 \%)$ & \\
\hline HPV+ & $49(26.7 \%)$ & $38(12.2 \%)$ & \\
\hline Missing & $1(0.5 \%)$ & $4(1.3 \%)$ & \\
\hline
\end{tabular}


Table 3 (on next page)

The hazard ratio of PLAU expression and clinical parameters in 496 HNSCC patients 
1 Table 3. The hazard ratio of PLAU expression and clinical parameters in 496 HNSCC 2 patients

Parameter

Univariate analysis

\begin{tabular}{lccc}
\cline { 3 - 4 } & HR & 95\%CI & P-value \\
\hline Age & & & $0.018^{*}$ \\
Older vs. Younger & 1.021 & $1.003 \sim 1.034$ &
\end{tabular}

\section{Gender}

Female vs. Male

0.728

$0.507 \sim 1.047$

0.087

\section{Clinical stage}

III-IV vs I-II

2.902

$1.605 \sim 5.25$

$<0.001 * * *$

Tumor size

T3+T4 vs. T1+T2

1.562

$1.158 \sim 2.109$

$0.003^{* *}$

Neck node metastasis

$$
\mathrm{N}+\text { vs. N- }
$$

$1.258 \sim 2.365$

$<0.001 * * *$

HPV

$$
\text { HPV+ vs. HPV- }
$$

0.468349

$0.312 \sim 0.703$

$$
<0.001 * * *
$$

\section{PLAU}

High vs. Low

1.667282

$1.218-2.282$

$0.001 * * *$ 